\title{
Forecasting the Impact of Stellar Activity on Transiting Exoplanet Spectra
}

\author{
Robert T. Zellem ${ }^{1}$, Mark R. Swain ${ }^{1}$, Gael Roudier ${ }^{1}$, Evgenya L. Shkolnik ${ }^{2}$, Michelle J. Creech-Eakman ${ }^{3}$, David R. Ciardi ${ }^{4}$, \\ Michael R. Line ${ }^{2}$, Aishwarya R. Iyer ${ }^{1}$, Geoffrey Bryden ${ }^{1}$, Joe Llama ${ }^{5}$, and Kristen A. Fahy ${ }^{1}$ \\ ${ }^{1}$ Jet Propulsion Laboratory, California Institute of Technology, 4800 Oak Grove Drive, Pasadena, CA 91109, USA; rzellem@jpl.nasa.gov \\ ${ }^{2}$ School of Earth \& Space Exploration, Arizona State University, Tempe, AZ 85281, USA \\ ${ }^{3}$ Department of Physics, New Mexico Institute of Mining and Technology, 801 Leroy Place, Socorro, NM 87801, USA \\ ${ }^{4}$ NASA Exoplanet Science Institute, California Institute of Technology, 1200 East California Boulevard, Pasadena, CA 91125, USA \\ ${ }^{5}$ Lowell Observatory, 1400 West Mars Hill Road, Flagstaff, AZ 86001, USA \\ Received 2017 May 10; revised 2017 June 5; accepted 2017 June 13; published 2017 July 19
}

\begin{abstract}
Exoplanet host star activity, in the form of unocculted starspots or faculae, alters the observed transmission and emission spectra of the exoplanet. This effect can be exacerbated when combining data from different epochs if the stellar photosphere varies between observations due to activity. Here, we present a method to characterize and correct for relative changes due to stellar activity by exploiting multi-epoch $\geqslant 2$ visits/transits) observations to place them in a consistent reference frame. Using measurements from portions of the planet's orbit where negligible planet transmission or emission can be assumed, we determine changes to the stellar spectral amplitude. With the analytical methods described here, we predict the impact of stellar variability on transit observations. Supplementing these forecasts with Kepler-measured stellar variabilities for F-, G-, K-, and M-dwarfs, and predicted transit precisions by the James Webb Space Telescope's (JWST) NIRISS, NIRCam, and MIRI, we conclude that stellar activity does not impact infrared transiting exoplanet observations of most presently known or predicted TESS targets by current or near-future platforms, such as JWST, as activity-induced spectral changes are below the measurement precision.
\end{abstract}

Key words: methods: analytical - planets and satellites: atmospheres - planets and satellites: detection stars: activity - starspots

\section{Introduction}

Stellar variability, in the form of starspots and faculae, can affect the measured transit depth of an exoplanet and hence its spectrum and retrieved physical properties (Pont et al. 2008; Silva-Valio 2008; Czesla et al. 2009; Wolter et al. 2009; Agol et al. 2010; Berta et al. 2011; Carter et al. 2011; Désert et al. 2011; Sing et al. 2011; Fraine et al. 2014; McCullough et al. 2014; Oshagh et al. 2014; Barstow et al. 2015; Damasso et al. 2015; Zellem et al. 2015; Rackham et al. 2017). As a worst-case scenario for very active stars, unocculted spots can cause an underestimation of the planet-to-star radius ratio of up to $4 \%$ at near-infrared wavelengths and up to $10 \%$ at visible wavelengths, while faculae can cause an overestimation of the planet-to-star radius ratio of up to $\sim 0.2 \%$ at near-infrared wavelengths and up to $\sim 3 \%$ at visible wavelengths (Oshagh et al. 2014). Unocculted spots can also mimic a Rayleigh scattering slope indicative of haze; for example, the visible and near-IR slope of the exoplanet HD 189733b's transit absorption spectrum, interpreted as Rayleigh scattering by haze particles (Pont et al. 2008, 2013; Sing et al. 2011, 2016), has also been interpreted as unocculted starspots on its active $\mathrm{K} 0$ host star (McCullough et al. 2014). Unocculted spots can also introduce false molecular spectral modulation into an exoplanet's spectrum, such as $\mathrm{H}_{2} \mathrm{O}$ if the spots are sufficiently cool (Fraine et al. 2014; Barstow et al. 2015). Stellar faculae, which are brighter than the stellar photosphere, decrease the transit depth at optical wavelengths (Rackham et al. 2017). Evolving unocculted spots on an active host star could also pose a problem when stitching data together from multiple epochs spanning multiple wavelengths, as will be required to completely sample an exoplanet from 0.6-28 $\mu \mathrm{m}$ with NASA's James Webb Space Telescope (JWST; Barstow et al. 2015). Therefore, spectroscopic observations of an exoplanet orbiting an active star have the potential to result in an erroneous interpretation of its atmospheric properties if the measurements have sufficient precision. As transit spectroscopic measurements become increasingly precise, especially in the JWST era, the possibility of contamination of the transit signal by starspots must be examined with care.

Fortunately, current transit spectroscopy, for example, with Hubble/WFC3, provides an opportunity to characterize exoplanet host stars as the out-of-transit and in-eclipse portions of the light curve probe emission from the host star alone, assuming negligible transmission and emission from the exoplanet. This method has the advantage that stellar activity can be quantified from the same data set used to measure the planet's transit or eclipse, assuming sufficient phase coverage. Extensive amounts of high-precision Hubble/WFC3 data are available for such an analysis (e.g., Iyer et al. 2016; Sing et al. 2016; Stevenson 2016; Tsiaras et al. 2017), which is interesting for both directly assessing the potential unocculted stellar activity contamination of existing measurements and as an independent check on conclusions about stellar activity based on ground-based monitoring.

Previous studies have characterized the stellar activity of exoplanet host stars via long-term, ground-based, visible, and near-infrared photometric (e.g., Pont et al. 2008, 2013; Berta et al. 2011; Désert et al. 2011; Sing et al. 2011; Knutson et al. 2012; Narita et al. 2013; Kreidberg et al. 2015; Nascimbeni et al. 2015; Zellem et al. 2015) and spectroscopic monitoring (e.g., Baliunas et al. 1995; Lovis et al. 2011; Robertson et al. 2013a, 2013b). However, ground-based observations are limited by the observability of the target during the year and weather, occasionally preventing them from being simultaneous with the space-based observations (e.g., Knutson et al. 2012). Non-contemporaneous observations make it difficult to determine the level of activity of the host star at the time of the 
space-based observations. Other studies have characterized changes in an exoplanet's transmission spectrum via detailed modeling of spectral modulation of the host star due to unocculted spots and plages (Oshagh et al. 2014; Rackham et al. 2017). Alternatively, we demonstrate here a method that uses the out-of-transit data itself to characterize stellar activity and its impact on the observed transiting exoplanet spectrum between epochs of observations.

As an illustrative example, we study here in detail the subNeptune GJ 1214b. This transiting exoplanet is one of the best sources to apply our analysis techniques as it has a relatively bright host star $(\mathrm{H}-\mathrm{mag}=9.094)$, providing high-precision measurements (signal-to-noise ratio $\approx 7000$ per pixel-based spectral channel, per orbit), and has been observed with 15 visits over 1 year (2012 September 27-2013 August 20) with Hubble/ WFC 3 and the G141 grism (1.15-1.65 $\mu \mathrm{m})$, allowing us to search for stellar variability and assess its impact on the observed exoplanet spectrum. With multiple Hubble visits, we quantify relative changes in the host star's spectrum with time, sensitive to the presentation, formation, and evolution of starspots and faculae.

Using the analytical expressions described here, Keplermeasured F-, G-, K-, and M-dwarf variabilities (D. R. Ciardi 2017, in preparation), and the projected precisions of JWST $\mathrm{s}$ MIRI, NIRCam, and NIRISS (Greene et al. 2016), we find that stellar variability does not impact a majority of transit observations for both currently known and projected Transiting Exoplanet Survey Satellite (TESS; Sullivan et al. 2015) targets. Thus, our study anticipates additional future, repeated high-precision spectroscopic transit measurements to observe stellar variations in exoplanet host stars such as with the New Mexico Exoplanet Spectroscopic Survey Instrument (NESSI; Jurgenson et al. 2010), which will commence a survey of $\sim 30$ transiting exoplanets on the 200 inch Hale Telescope at Palomar Observatory within the next year; ESA's Atmospheric Remote-sensing Exoplanet Large-survey (ARIEL), a proposed dedicated transiting exoplanet survey that will repeatedly measure the spectra $(\sim 2-8 \mu \mathrm{m})$ of hundreds of exoplanets with multiple visits (Puig et al. 2016; Tinetti et al. 2016); and JWST, which will measure the infrared spectra of tens of transiting exoplanets (Cowan et al. 2015; Stevenson et al. 2016).

\section{The Impact of Unocculted Stellar Activity on Transit Spectroscopy}

Unocculted starspots and faculae affect the observed planetary transmission spectrum via the equation (McCullough et al. 2014)

$$
\begin{aligned}
\delta_{\text {active,transit }}= & \underbrace{\left(\frac{R_{\text {planet }}}{R_{\text {star }}}\right)^{2}}_{\delta_{\text {quiescent, transit }}} \\
& \cdot\left[1+\left(\frac{R_{\text {feature }}}{R_{\text {star }}}\right)^{2}\left(\frac{B_{\text {feature }}}{B_{\text {star }}}-1\right)\right]^{-1},
\end{aligned}
$$

where $\delta_{\text {active,transit }}$ is the observed transmission spectrum modulated by unocculted stellar activity (spots or faculae); $\delta_{\text {quiescent,transit }}$ is the observed transmission spectrum when the star is in a quiescent state; $R_{\text {planet }}$ and $R_{\text {star }}$ are the radii of the exoplanet and host star, respectively; and $B_{\text {star }}$ is the spectrum of the star. $R_{\text {feature }}$ is the effective radius of the features (spots or faculae), if all features visible on the surface of the star were to be combined into one large complex. $B_{\text {feature }}$ is the spectrum of this complex. Please note that all of these variables are functions of wavelength; we have omitted the wavelength symbol $\lambda$ for clarity.

The impact of stellar activity on eclipse measurements is derived as follows. For an inactive star, the measured "quiescent" eclipse depth is

$$
\begin{aligned}
\delta_{\text {quiescent,eclipse }}= & \frac{F_{\text {out-of-eclipse }}-F_{\text {in-eclipse }}}{F_{\text {in-eclipse }}} \\
= & \frac{F_{\text {in-eclipse }}+B_{\text {planet }} R_{\text {planet }}^{2}-F_{\text {in-eclipse }}}{F_{\text {in-eclipse }}} \\
& =\frac{B_{\text {planet }} R_{\text {planet }}^{2}}{B_{\text {star }} R_{\text {star }}^{2}} .
\end{aligned}
$$

Whereas for an active star, the observed eclipse depth is

$$
\begin{aligned}
& \delta_{\text {active,eclipse }}=\frac{F_{\text {out-of-eclipse }}-F_{\text {in-eclipse }}}{F_{\text {in-eclipse }}} \\
& =\frac{F_{\text {in-eclipse }}+B_{\text {planet }} R_{\text {planet }}^{2}-F_{\text {in-eclipse }}}{F_{\text {in-eclipse }}} \\
& =\frac{B_{\text {planet }} R_{\text {planet }}^{2}}{B_{\text {star }} R_{\text {star }}^{2}+B_{\text {feature }} R_{\text {feature }}^{2}-B_{\text {star }} R_{\text {feature }}^{2}} \\
& =\frac{B_{\text {planet }} R_{\text {planet }}^{2}}{B_{\text {star }} R_{\text {star }}^{2} \cdot\left(1+\frac{B_{\text {feature }} R_{\text {teature }}^{2}}{B_{\text {star }} R_{\text {star }}^{2}}-\frac{B_{\text {star }} R_{\text {teature }}^{2}}{B_{\text {star }} R_{\text {star }}^{2}}\right)} \\
& =\underbrace{\frac{B_{\text {planet }} R_{\text {planet }}^{2}}{B_{\text {star }} R_{\text {star }}^{2}}}_{\delta_{\text {quiescent, eclipse }}} \\
& \cdot\left[1+\left(\frac{R_{\text {feature }}}{R_{\text {star }}}\right)^{2}\left(\frac{B_{\text {feature }}}{B_{\text {star }}}-1\right)\right]^{-1} .
\end{aligned}
$$

Note that the equation for the active-star eclipse depth $\delta_{\text {active,eclipse }}$ (Equation (3)) is the same as for transit $\delta_{\text {active,transit }}$ (Equation (1)).

We then isolate and define the "stellar activity correction factor" $\zeta$, a wavelength-dependent function that describes how much unocculted spots and faculae alter the planetary transit depths from their true, inactive values:

$$
\zeta=1+\left(\frac{R_{\text {feature }}}{R_{\text {star }}}\right)^{2}\left(\frac{B_{\text {feature }}}{B_{\text {star }}}-1\right) .
$$

We can then rearrange Equation (4) as

$$
\begin{aligned}
\zeta= & 1+\left(\frac{R_{\text {feature }}}{R_{\text {star }}}\right)^{2}\left(\frac{B_{\text {feature }}}{B_{\text {star }}}-1\right) \\
= & \frac{1}{B_{\text {star }} R_{\text {star }}^{2}}\left[B_{\text {star }} R_{\text {star }}^{2}+B_{\text {star }} R_{\text {feature }}^{2}\left(\frac{B_{\text {feature }}}{B_{\text {star }}}-1\right)\right] \\
= & \frac{1}{B_{\text {star }} R_{\text {star }}^{2}}\left[B_{\text {star }} R_{\text {star }}^{2}+B_{\text {feature }} R_{\text {feature }}^{2}\right. \\
& \left.-B_{\text {star }} R_{\text {feature }}^{2}\right] \\
= & \frac{F_{\text {active }}}{F_{\text {quiescent }}}
\end{aligned}
$$


where $F_{\text {active }}$ is the measured spectrum of the active star and $F_{\text {quiescent }}$ is the spectrum of the quiescent star.

By combining Equations (1) and (3) with (4) and (5), we find

$$
\begin{aligned}
\delta_{\text {active }} & =\delta_{\text {quiescent }} \cdot \zeta^{-1} \\
\delta_{\text {active }} & =\delta_{\text {quiescent }} \frac{F_{\text {quiescent }}}{F_{\text {active }}} \\
\frac{\delta_{\text {active }}}{\delta_{\text {quiescent }}} & =\frac{F_{\text {quiescent }}}{F_{\text {active }}} .
\end{aligned}
$$

Thus, the measured transit/eclipse depth is inversely proportional to the brightness of the star. For example, if the star decreases in brightness by $1 \%$ due to an unocculted spot, then the transit/eclipse depth will deepen by $1 \%$, and vice versa. Thus, by observing the spectrum of the star when it is quiescent $F_{\text {quiescent }}$, one can use Equation (6) to correct the observed exoplanet's transmission or emission spectrum for unocculted stellar activity.

This equation is powerful as it allows one to observe exoplanets of interest that have the misfortune of having a variable host star. By observing the system at least twice, one can measure the spectra of the host star $F$ with the out-of-transit and in-eclipse portions of the light curve to quantify $\zeta$ (Section 3.1) and then correct for the relative changes in the observed planetary spectra due to unocculted stellar activity with Equation (6) to place the observations in a consistent reference frame.

However, with a limited number of repeat visits, determining which epoch(s) are active or quiescent is not necessarily straightforward. One could implement stellar models (e.g., PHOENIX; Husser et al. 2013) to determine which epoch(s) agree with the stellar model, indicating quiescence $F_{\text {quiescent }}$. Alternatively, one could use ground-based monitoring or multiple visits (e.g., GJ 1214 features 15 visits with Hubble/ WFC3; see Section 3) to monitor stellar changes. At the very least, one can use the measured stellar variability either with the data itself (Section 3.1), with previous observations of typical stellar activities (Section 4; e.g., D. R. Ciardi 2017, in preparation), or with ground-based monitoring to estimate the change in the exoplanet's observed transit depth $\Delta \delta$ :

$$
\begin{aligned}
\Delta \delta & =\delta_{\text {active }}-\delta_{\text {quiescent }} \\
& =\delta_{\text {quiescent }} \frac{F_{\text {quiescent }}}{F_{\text {active }}}-\delta_{\text {quiescent }} \\
& =\delta_{\text {quiescent }}\left(\frac{F_{\text {quiescent }}}{F_{\text {active }}}-1\right) \\
& =\delta_{\text {quiescent }}\left(\frac{F_{\text {quiescent }}-F_{\text {active }}}{F_{\text {active }}}\right) .
\end{aligned}
$$

Thus, for example, if a planet with a $1 \%$ transit depth orbits a star that is active at the $0.1 \%$ level, then the planet's observed transit depth will change by $10 \mathrm{ppm}$. Therefore, by knowing the typical activity of a host star, one can predict the relative change in the observed transit depth $\Delta \delta$ to determine if it is larger than the precision of their data and it would adversely impact their observations (Section 4). However, if these additional sources of information are unavailable, then one can at the very least place all of the transit observations into a consistent reference frame.

\section{GJ 1214: An Example Variable System in the Hubble/WFC3 Bandpass}

We demonstrate this stellar characterization method with 14 visits of Hubble/WFC3 spectroscopy $(1.15-1.65 \mu \mathrm{m})$ of GJ 1214 spanning 2012 September 27-2013 August 20 (PID: 13021, PI: Jacob Bean). These data form the basis for the published spectrum of the sub-Neptune GJ 1214b, which was found to be flat, suggesting high-altitude clouds (Kreidberg et al. 2014a). The exoplanet host star GJ 1214 is a nearby (14.6 pc = 47.5 ly; Anglada-Escudé et al. 2013) M4.5V dwarf star (Lurie et al. 2014). It is metal-rich, with a spectroscopically derived $[\mathrm{Fe} / \mathrm{H}]$ ranging from 0.26 to 0.39 (Rojas-Ayala et al. 2010; Anglada-Escudé et al. 2013; Newton et al. 2015). GJ 1214 is $3-10$ Gyr old (Reid \& Gizis 2005; Charbonneau et al. 2009); at these older ages, stellar activity is expected to be fairly low, which is generally consistent with Hubble/STIS observations of the system that show little to no Ly $\alpha$ emission (France et al. 2013).

Despite this finding, multiple studies have determined that GJ 1214 is active due to both starspots and faculae (Charbonneau et al. 2009; Berta et al. 2011; Carter et al. 2011; Kundurthy et al. 2011; Fraine et al. 2013; Kreidberg et al. 2014a; Rackham et al. 2017). GJ 1214's variability is not unexpected: for those M-dwarfs that are located $\leqslant 25 \mathrm{pc}$ away, $50 \% \pm 25 \%$ of M4 stars are active for $4.5_{-1.0}^{+0.5} \mathrm{Gyr}$ and $90 \% \%_{-17.5 \%}^{+2.5 \%}$ of M5 stars are active for $7.0 \pm 0.5 \mathrm{Gyr}$ (West et al. 2008). As such, multiple groups have conducted large ground-based photometric campaigns to characterize GJ 1214's long-term stellar variability. For example, the MEarth survey $(40 \mathrm{~cm}$ telescope; Sloan $i+z$ photometry) found that GJ 1214 was active during the spring observing seasons in 2008, 2009, and 2010 with a semi-amplitude $A=3.5 \pm 0.7 \mathrm{mmag}$ and a period of 53 days and V-band photometry with the $1.2 \mathrm{~m}$ FLWO/KeplerCam at Mt. Hopkins, AZ, from 2010 March 26 to 2010 June 17 measured $A=7 \pm 3 \mathrm{mmag}$ and a period of 41 days (Berta et al. 2011). A large 32 night photometric campaign spanning 78 nights in 2012 with the $50 \mathrm{~cm}$ MISuME telescope determined that GJ 1214 was active with a period of $44.3 \pm 1.2$ days with $A=$ $3.47 \pm 0.41 \mathrm{mmag}$ in the $I_{c}$ band, $A=0.61 \pm 0.04 \mathrm{mmag}$ in the $R_{c}$ band, and $A=2.34 \pm 0.46$ mmag in the $g^{\prime}$ band (Narita et al. 2013). A 191 night multi-band photometric campaign (110 nights from 2012 March 21 to October 10 and 81 nights from 2013 March 24 to October 4) with the $1.2 \mathrm{~m}$ STELLA/WiFSIP found that GJ 1214 was active in the $V_{J}$ band from 2012 to 2013 with $A=11.4 \pm 1.1 \mathrm{mmag}$ and a period of $83.0 \pm 0.8$ days, in the $V_{J}$ band in 2012 with $A=15.1 \pm 1.3 \mathrm{mmag}$ and a period of $69.0 \pm$ 2.0 days, in the $I_{c}$ band in 2012 with $A=12.0 \pm 1.2 \mathrm{mmag}$ and a period of $79.6 \pm 2.5$ days, and in the $B_{J}$ band in 2012 with $A \geqslant 15 \mathrm{mmag}$ and a period of $\geqslant 70$ days (Nascimbeni et al. 2015). For comparison, based on a study of Kepler observations $(0.42-0.9 \mu \mathrm{m})$, M-dwarfs have a median activity level of $6.83 \mathrm{mmag}$ over a baseline of 100 days (D. R. Ciardi 2017, in preparation). Thus compared to other M-dwarfs, GJ 1214's variability is typical.

To further investigate stellar variability with a previously unused resource, we characterize GJ 1214's stellar activity with the out-of-transit portions of the Hubble/WFC3 data itself. Then, using the formulae previously defined in Section 2, we quantify the effects of GJ 1214's stellar variability on the exoplanet's measured transmission spectrum. 


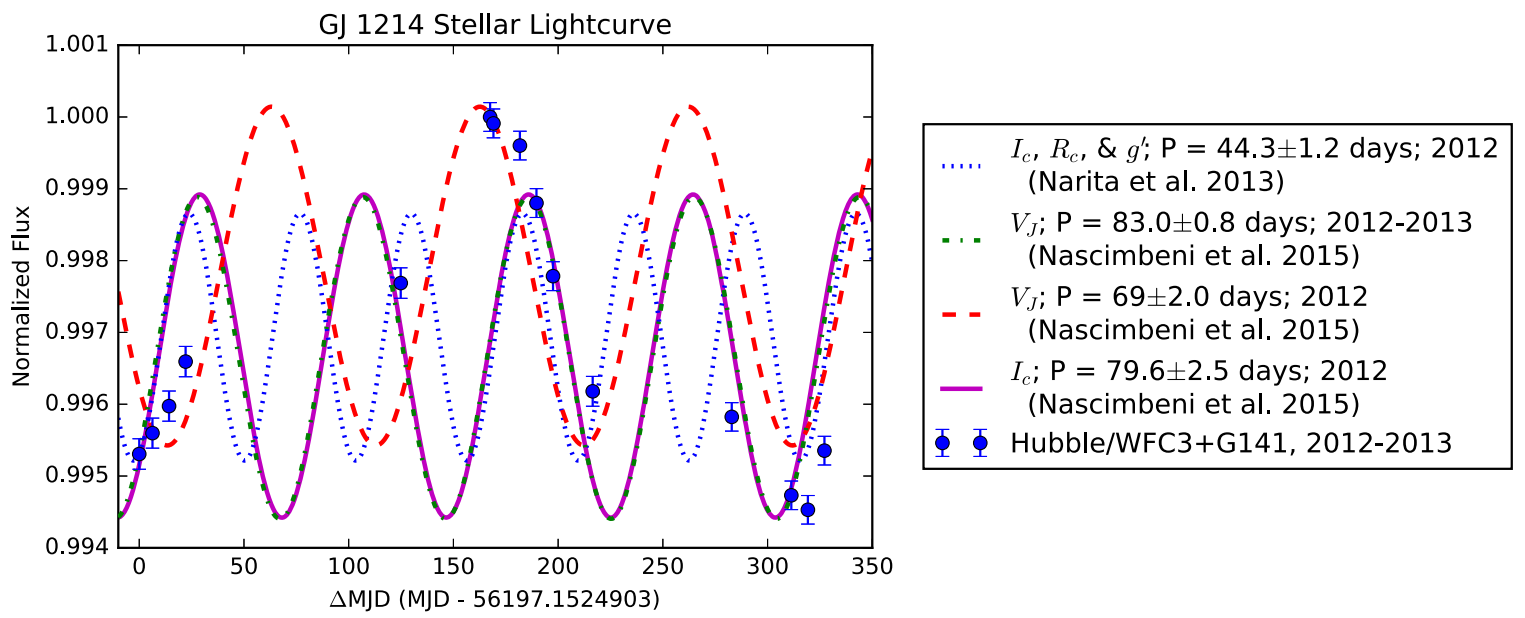

Figure 1. GJ 1214: an exoplanet host star with amplitude variability. GJ 1214's stellar light curve (blue circles), integrated over the Hubble/WFC3+G141 passband, indicates a semi-amplitude variability of $A=0.279 \% \pm 0.012 \%=2.34 \pm 0.46 \mathrm{mmag}$, which is $3.77 \times$ smaller than semi-amplitudes measured in the optical over a similar time-frame (Nascimbeni et al. 2015). In addition, the near-infrared period $P$ of GJ 1214's variability, when fitting for the amplitude and phase offset, is incompatible with previously reported values measured at shorter wavelengths (dotted, dashed-dotted, dashed, and solid lines), potentially due to evolution of the activity.

\subsection{Data Extraction and Analysis}

We extract GJ 1214's time-varying Hubble/WFC3 spectra from the STScI-calibrated ima files (Dressel 2017) by taking the difference between non-destructive reads to provide a background subtraction, wavelength-calibrating the spectra with a G141 filter transmission curve, flagging bad pixels, and taking the mean along the spatial scan axis. We then remove the first orbit of each visit to mitigate Hubble/WFC3's "ramp effect" (Berta et al. 2012; Swain et al. 2013; Kreidberg et al. 2014a, 2014b; Stevenson et al. 2014), stack and order the spectra in time, isolate the out-of-transit portions, and sum over all wavelengths to construct GJ 1214's broadband stellar light curve (Figure 1).

We find that GJ 1214 has a near-infrared semi-amplitude $A=0.279 \% \pm 0.012 \%=2.34 \pm 0.46 \mathrm{mmag}$, which is $3.77 \times$ smaller than its visible wavelength $\left(V_{J}\right.$ photometric band) semi-amplitude of $11.4 \pm 1.1 \mathrm{mmag}$ measured over a similar time-frame (Nascimbeni et al. 2015). We then fit GJ 1214's time-varying flux with multiple sine waves using a Markov Chain Monte Carlo (MCMC; e.g., Ford 2005) and adopting as priors previously reported periods ( $44.3 \pm 1.2$ days, Narita et al. 2013; $83.0 \pm 0.8,69.0 \pm 2.0$ and $79.6 \pm 2.5$ days, Nascimbeni et al. 2015). However, none of these periods, measured at shorter wavelengths, fits all of our near-infrared data (Figure 1), including the period of $83.0 \pm 0.8$ days, measured in the $V_{J}$ band over a similar time-frame as the Hubble/WFC3 observations (Nascimbeni et al. 2015). This discrepancy is potentially due to the fact that these visible-band data (2012 March 21-October 10; 2013 March 24-October 4) were not fully contemporaneous with the Hubble/WFC3 observations (2012 September 27-October 19; 2013 January 30-May 1; 2013 July 7-August 20). Thus, GJ 1214's spots or faculae could have evolved on its surface, changing its apparent period, thereby rendering our near-infrared result incompatible with its visible-band period. However, despite this apparent spot evolution, our data agree and complement to first order GJ 1214's $2013 V_{J}$ and $B_{J}$ photometry (Nascimbeni et al. 2015).

\subsection{Assessing the Impact of Stellar Activity on Planetary Spectra}

We next quantify the effect of this stellar activity on the observed per-visit GJ 1214b spectra. Because only a final visitaveraged spectrum was published for GJ 1214b (Kreidberg et al. 2014a), we first estimate the planet's per-visit spectra by increasing the uncertainties on the published spectra by the square root of the number of visits (14). Since we explore how much the planetary spectrum could change due to unocculted activity, we invert Equation (6) to solve for the active-star planetary spectrum $\delta_{\text {active }}$ for each visit and use the mean stellar spectrum as a reference in place of GJ 1214's quiescent spectrum $\delta_{\text {quiescent }}$.

We find that for most spectral channels, stellar activity does not measurably modulate the planet's per-visit spectrum (Figure 2, top). This outcome is even more clear when we bin the per-visit spectra in time (Figure 2, middle and bottom). Therefore, merely using only the Hubble/WFC3 data themselves, we confirm the assessment of Kreidberg et al. (2014a), which was based on simulations of stellar activity, that GJ 1214b's spectrum is not statistically impacted by stellar variability at this level of measurement precision $(\sim 30 \mathrm{ppm})$.

\section{Forecasting the Effect of Stellar Activity on Other Instruments}

Using Equation (7), we forecast the change in an exoplanet's observed transit depth as a function of its transit depth and host star activity (Figure 3). Our measurement of GJ 1214's variability (magenta star) is included for reference. We also plot median stellar variabilities measured by Kepler over a 25 day period for F-, G-, K-, and M-dwarfs (D. R. Ciardi 2017, in preparation). We chose a 25 day timescale as it is similar to the amount of time TESS will observe each of its fields (27.4 days; Ricker et al. 2014). Thus, one can use the stellar variability measured by TESS and Figure 3 to forecast if their transit measurements will be impacted by stellar activity. However, since mid- to late-M-dwarf stars typically have longer rotation periods ( $\sim 100$ days; Newton et al. 2016), this 

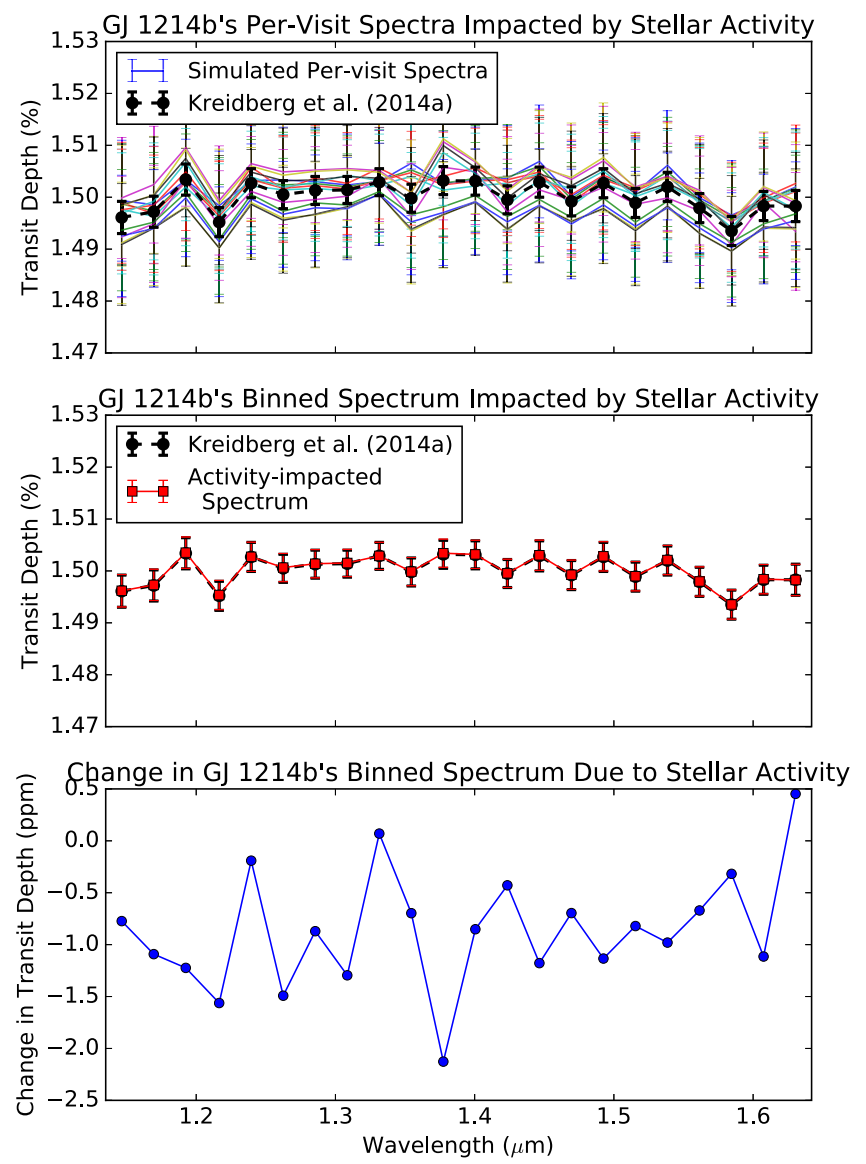

Figure 2. Stellar activity does not affect GJ 1214b's spectrum. Top panel: the visit-to-visit changes in the exoplanet's measured transmission spectrum due to stellar activity (thin, multi-colored lines) compared to GJ 1214b's visit-binned published spectra (black circles; Kreidberg et al. 2014a). Middle panel: transmission spectrum constructed by averaging the stellar activity modulated per-visit spectra (red squares) compared to the published transmission spectrum (black circles). Bottom panel: differential spectrum of the published transmission spectrum and the stellar activity modulated spectrum. Stellar activity does not statistically alter the exoplanet's measured spectrum.

25 day timescale may underestimate their variability. When using a 100 day timescale, we find that M-dwarfs have a median variability that is $1.3 \times$ larger than the 25 day timescale (D. R. Ciardi 2017, in preparation). We have omitted plotting the M-dwarf 100 day median variability on Figure 3 for clarity.

Stellar activity at visible wavelengths measured by Kepler are important because they provide a "worst-case" scenario for infrared transit measurements as stellar activity has a larger impact on the observed transit depth in the visible than the infrared (Oshagh et al. 2014; Rackham et al. 2017). Therefore, using Figure 3, one can forecast the impact of stellar variability of their infrared observations on any platform given their target's transit depth and host star spectral type.

We also include the projected measurement precisions of a 6.37 H-mag transiting exoplanet system with JWST's NIRISS, NIRCam, and MIRI (Greene et al. 2016). This magnitude limit is appropriate for guiding the next few years of transiting exoplanet observations as nearly all of the top 1200 targets are dimmer than $6 \mathrm{H}$-mag (Figure 4), when including both currently known and predicted TESS yields (Sullivan et al. 2015) ranked by a

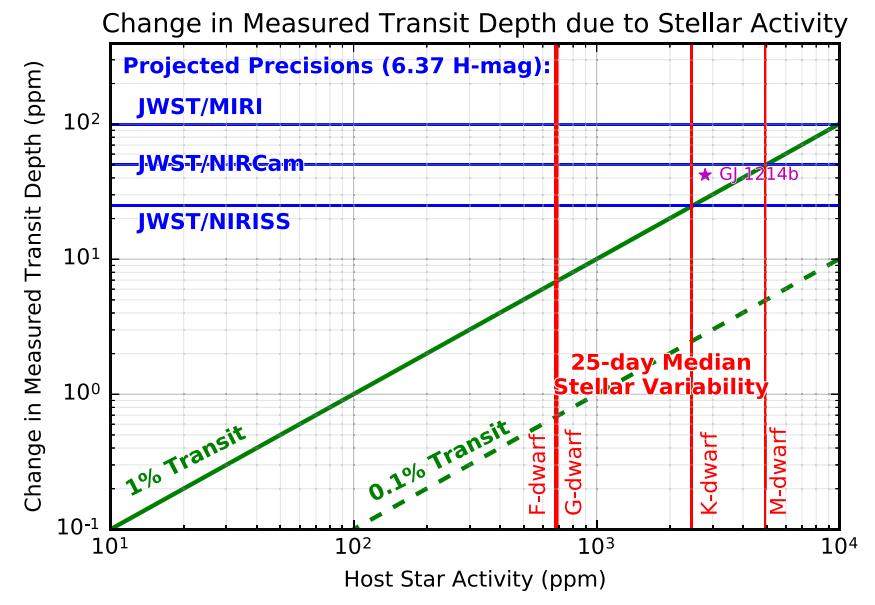

Figure 3. Host star activity minimally impacts infrared transit observations. Forecasted change in the exoplanet's measured transit depth as a function of its transit depth (green diagonal lines; Equation (7)) and host star's activity. Also shown are the 25 day median stellar variabilities of F-, G-, K-, and M-dwarf stars as measured by Kepler (red vertical lines; D. R. Ciardi 2017, in preparation); these variabilities provide a conservatively high estimate as the effects of stellar activity on transit observations are more pronounced in the visible than in the infrared (Oshagh et al. 2014; Rackham et al. 2017). Also included are the projected transit precisions of JWST's NIRISS, NIRCam, and MIRI observations of an exoplanet with a $6.37 \mathrm{H}$-mag host star (blue horizontal lines; Greene et al. 2016) and GJ 1214's stellar variability measured in this study (magenta star; Figure 1). We predict here that infrared observations by JWST, and therefore most present and near-future platforms, will largely be unaffected by stellar variability.

platform-independent figure of merit (FOM):

$$
\begin{gathered}
\text { FOM }=\frac{\text { signal }}{\text { noise }}, \\
=\frac{1 \mathrm{H}_{s} \text { of spectral modulation }}{\text { photon noise }}, \\
=\frac{2 H_{s} R_{p} R_{s}^{-2}}{10^{0.2 H-\mathrm{mag}}},
\end{gathered}
$$

where $H$-mag is the host star's apparent magnitude in the H-band and $H_{s}$ is an exoplanet's scale height:

$$
H_{s}=\frac{k_{\mathrm{B}} T_{e q}}{\mu g},
$$

where $k_{\mathrm{B}}$ is the Boltzmann constant, $T_{\mathrm{eq}}$ is the planet's equilibrium temperature, $\mu$ is the planet's mean molecular weight, and $g$ is the planet's acceleration due to gravity. The values for $T_{\text {eq }}$ and $g$ are derived from parameters published in NASA's Exoplanet Archive (Akeson et al. 2013). However, because most of the targets in the 1200 planet sample lack detailed spectroscopic measurements, their atmospheric compositions are unknown and therefore must be selected randomly from some prescribed distribution to estimate their mean molecular weight $\mu$. Therefore, we assign a metallicity to each planet based on an assumed relationship where metallicity increases as planet mass decreases. This relationship is motivated both by simulations of planet formation (Fortney et al. 2013) and by observations of methane in the solar system's giant planets (Wong et al. 2004; Fletcher et al. 2009; Karkoschka \& Tomasko 2011; Sromovsky et al. 2011). The 


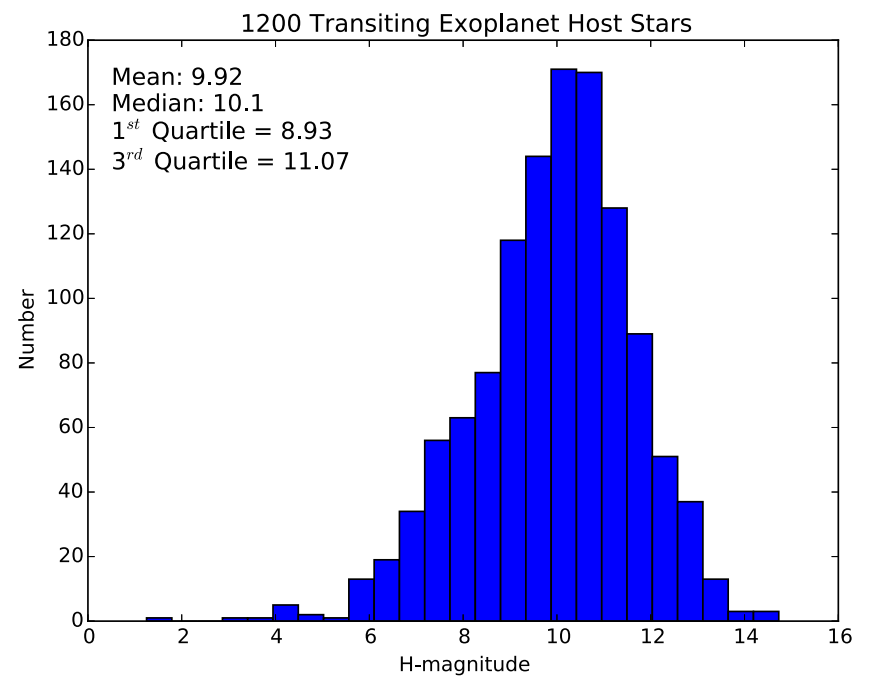

Figure 4. The 1200 best targets for transiting exoplanet absorption spectroscopy. These targets, identified with a transit figure of merit (Equation (10)), contain both presently known targets and forecasted targets discovered by TESS (Sullivan et al. 2015) and therefore represent the most likely targets of, for example, JWST.

planet and star in each system are assigned the same $\mathrm{C} / \mathrm{O}$ ratio, drawn from a Gaussian distribution with a mean $\log [\mathrm{C} / \mathrm{O}]$ of -0.2 and a dispersion of 0.12 (cf. -0.26 for the Sun). Our choice for the $\mathrm{C} / \mathrm{O}$ distribution results in $5 \%$ of systems having $\mathrm{C} / \mathrm{O}>1$, which is within the range of estimates from stellar observations (Fortney 2012; Hinkel et al. 2014). Given the metallicity and $\mathrm{C} / \mathrm{O}$ ratio assigned to each planet, we calculate the full range of molecular abundances within its atmosphere assuming equilibrium chemistry with the CEA code (McBride \& Gordon 1996). We then translate these abundances into an overall mean molecular weight $\mu$ for each atmosphere.

We find that most infrared transit observations by JWST (Cowan et al. 2015; Stevenson et al. 2016) and other current and near-future platforms, such as NESSI (Jurgenson et al. 2010) and ARIEL (Puig et al. 2016), are unaffected by stellar variability, except for rare cases where the target is bright, relatively active, and has a large transit depth (e.g., HD 189733 b or, alternatively, targets with a $\sim 1 \%$ transit depth orbiting bright M-dwarfs). This conclusion is qualitatively in agreement with a study of the effects of stellar variability on the atmospheric retrievals of JWST transit spectroscopy observed at different epochs and observing modes (Barstow et al. 2015).

\section{Conclusions and Future Work}

Here, we analytically derive the change in an exoplanet's transit depth as a function of host star activity and provide a method for mitigating stellar activity on multi-epoch $(\geqslant 2$ visits) transit observations by characterizing the activity of the host star from the out-of-transit and in-eclipse portions of the light curve. An advantage of this technique is that it does not require modeling of the stellar activity itself to place them in a consistent reference frame; however, incorporating unocculted spot and faculae modeling (Oshagh et al. 2014; Rackham et al. 2017) in conjunction with the techniques presented here would be beneficial, as it has the potential to identify the star's quiescent state and is subject to future work. Using Kepler observations of stellar variability for a variety of stellar spectral types and predicted precisions for JWST instruments, we conclude that infrared observations of currently known and TESS-discovered transiting exoplanets by present and nearfuture platforms, such as JWST, are largely unaffected by stellar variability. However, certain highly variable stars (e.g., HD 189733 or bright M-dwarfs with large, $\gtrsim 1 \%$, transit depths) may still need special consideration, and a first-order spectral correction can be implemented with the formalisms presented here (Section 2).

Using the methods provided here, we analyze publicly available Hubble/WFC3 transit spectroscopy of GJ 1214 and determine that it is active with a semi-amplitude of $0.279 \% \pm 0.012 \%=3.02 \pm 1.30 \mathrm{mmag}, 3.77 \times$ smaller than its measured activity at visible wavelengths over a similar time $(11.4 \pm 1.1 \mathrm{mmag}$; Nascimbeni et al. 2015). We also find that GJ 1214's visible-band periods are incompatible with its nearinfrared data, potentially due to stellar activity evolution occurring between the observations. Regardless, we confirm that GJ 1214's stellar activity does not statistically impact GJ 1214b's Hubble/WFC3+G141 near-infrared spectrum.

Part of the research was carried out at the Jet Propulsion Laboratory, California Institute of Technology, under contract with the National Aeronautics and Space Administration.

R.T.Z. would like to thank JPL's ExoSpec team and Jacob Bean for their insightful comments.

We thank the referee for helpful comments.

This research has made use of the NASA Exoplanet Archive, which is operated by the California Institute of Technology, under contract with the National Aeronautics and Space Administration under the Exoplanet Exploration Program. We thank the JPL Exoplanet Science Initiative for partial support of this work.

\section{References}

Agol, E., Cowan, N. B., Knutson, H. A., et al. 2010, ApJ, 721, 1861 Akeson, R. L., Chen, X., Ciardi, D., et al. 2013, PASP, 125, 989

Anglada-Escudé, G., Rojas-Ayala, B., Boss, A. P., Weinberger, A. J., \& Lloyd, J. P. 2013, A\&A, 551, A48

Baliunas, S. L., Donahue, R. A., Soon, W. H., et al. 1995, ApJ, 438, 269

Barstow, J. K., Aigrain, S., Irwin, P. G. J., Kendrew, S., \& Fletcher, L. N. 2015, MNRAS, 448, 2546

Berta, Z. K., Charbonneau, D., Bean, J., et al. 2011, ApJ, 736, 12

Berta, Z. K., Charbonneau, D., Désert, J.-M., et al. 2012, ApJ, 747, 35

Carter, J. A., Winn, J. N., Holman, M. J., et al. 2011, ApJ, 730, 82

Charbonneau, D., Berta, Z. K., Irwin, J., et al. 2009, Natur, 462, 891

Cowan, N. B., Greene, T., Angerhausen, D., et al. 2015, PASP, 127, 311

Czesla, S., Huber, K. F., Wolter, U., Schröter, S., \& Schmitt, J. H. M. M. 2009, A\&A, 505, 1277

Damasso, M., Biazzo, K., Bonomo, A. S., et al. 2015, A\&A, 575, A111

Désert, J.-M., Charbonneau, D., Demory, B.-O., et al. 2011, ApJS, 197, 14

Dressel, L. 2017, Wide Field Camera 3 Instrument Handbook, Version 9.0 (Baltimore, MD: STScI)

Fletcher, L. N., Orton, G. S., Teanby, N. A., Irwin, P. G. J., \& Bjoraker, G. L. 2009, Icar, 199, 351

Ford, E. B. 2005, AJ, 129, 1706

Fortney, J. J. 2012, ApJL, 747, L27

Fortney, J. J., Mordasini, C., Nettelmann, N., et al. 2013, ApJ, 775, 80

Fraine, J., Deming, D., Benneke, B., et al. 2014, Natur, 513, 526

Fraine, J. D., Deming, D., Gillon, M., et al. 2013, ApJ, 765, 127

France, K., Froning, C. S., Linsky, J. L., et al. 2013, ApJ, 763, 149

Greene, T. P., Line, M. R., Montero, C., et al. 2016, ApJ, 817, 17

Hinkel, N. R., Timmes, F. X., Young, P. A., Pagano, M. D., \& Turnbull, M. C. 2014, AJ, 148, 54

Husser, T.-O., Wende-von Berg, S., Dreizler, S., et al. 2013, A\&A, 553, A6

Iyer, A. R., Swain, M. R., Zellem, R. T., et al. 2016, ApJ, 823, 109

Jurgenson, C., Santoro, F., Creech-Eakman, M., et al. 2010, Proc. SPIE, 7735, 773519 
Karkoschka, E., \& Tomasko, M. G. 2011, Icar, 211, 780

Knutson, H. A., Lewis, N., Fortney, J. J., et al. 2012, ApJ, 754, 22

Kreidberg, L., Bean, J. L., Désert, J.-M., et al. 2014a, Natur, 505, 69

Kreidberg, L., Bean, J. L., Désert, J.-M., et al. 2014b, ApJL, 793, L27

Kreidberg, L., Line, M. R., Bean, J. L., et al. 2015, ApJ, 814, 66

Kundurthy, P., Agol, E., Becker, A. C., et al. 2011, ApJ, 731, 123

Lovis, C., Dumusque, X., Santos, N. C., et al. 2011, arXiv:1107.5325

Lurie, J. C., Henry, T. J., Jao, W.-C., et al. 2014, AJ, 148, 91

McBride, B. J., \& Gordon, S. 1996, Computer Program for Calculation of Complex Chemical Equilibrium Compositions and Applications: II. Users Manual and Program Description, NASA Ref. Publ. 1311 (Cleveland, OH: NASA Lewis Research Center)

McCullough, P. R., Crouzet, N., Deming, D., \& Madhusudhan, N. 2014, ApJ, 791, 55

Narita, N., Fukui, A., Ikoma, M., et al. 2013, ApJ, 773, 144

Nascimbeni, V., Mallonn, M., Scandariato, G., et al. 2015, A\&A, 579, A113

Newton, E. R., Charbonneau, D., Irwin, J., \& Mann, A. W. 2015, ApJ, 800, 85

Newton, E. R., Irwin, J., Charbonneau, D., Berta-Thompson, Z. K., \& Dittmann, J. A. 2016, ApJL, 821, L19

Oshagh, M., Santos, N. C., Ehrenreich, D., et al. 2014, A\&A, 568, A99

Pont, F., Knutson, H., Gilliland, R. L., Moutou, C., \& Charbonneau, D. 2008, MNRAS, 385, 109

Pont, F., Sing, D. K., Gibson, N. P., et al. 2013, MNRAS, 432, 2917

Puig, L., Pilbratt, G. L., Heske, A., Escudero Sanz, I., \& Crouzet, P.-E. 2016, Proc. SPIE, 9904, 99041W

Rackham, B., Espinoza, N., Apai, D., et al. 2017, ApJ, 834, 151
Reid, I. N., \& Gizis, J. E. 2005, PASP, 117, 676

Ricker, G. R., Winn, J. N., Vanderspek, R., et al. 2014, Proc. SPIE, 9143, 914320

Robertson, P., Endl, M., Cochran, W. D., \& Dodson-Robinson, S. E. 2013a, ApJ, 764, 3

Robertson, P., Endl, M., Cochran, W. D., MacQueen, P. J., \& Boss, A. P. 2013b, ApJ, 774, 147

Rojas-Ayala, B., Covey, K. R., Muirhead, P. S., \& Lloyd, J. P. 2010, ApJL, 720, L113

Silva-Valio, A. 2008, ApJL, 683, L179

Sing, D. K., Fortney, J. J., Nikolov, N., et al. 2016, Natur, 529, 59

Sing, D. K., Pont, F., Aigrain, S., et al. 2011, MNRAS, 416, 1443

Sromovsky, L. A., Fry, P. M., \& Kim, J. H. 2011, Icar, 215, 292

Stevenson, K. B. 2016, ApJL, 817, L16

Stevenson, K. B., Desert, J.-M., Line, M. R., et al. 2014, Sci, 346, 838

Stevenson, K. B., Lewis, N. K., Bean, J. L., et al. 2016, PASP, 128, 094401

Sullivan, P. W., Winn, J. N., Berta-Thompson, Z. K., et al. 2015, ApJ, 809 77

Swain, M., Deroo, P., Tinetti, G., et al. 2013, Icar, 225, 432

Tinetti, G., Drossart, P., Eccleston, P., et al. 2016, Proc. SPIE, 9904, 99041X

Tsiaras, A., Waldmann, I. P., Zingales, T., et al. 2017, arXiv:1704.05413

West, A. A., Hawley, S. L., Bochanski, J. J., et al. 2008, AJ, 135, 785

Wolter, U., Schmitt, J. H. M. M., Huber, K. F., et al. 2009, A\&A, 504, 561

Wong, M. H., Mahaffy, P. R., Atreya, S. K., Niemann, H. B., \& Owen, T. C. 2004, Icar, 171, 153

Zellem, R. T., Griffith, C. A., Pearson, K. A., et al. 2015, ApJ, 810, 11 